\title{
Home-based rehabilitation system for stroke survivors: a clinical evaluation
}

\author{
Enjie Ghorbel • Renato Baptista • \\ Abdelrahman Shabayek · Djamila \\ Aouada - Maialen Gorostiza Oramaeche . \\ Janire Orcajo Lago · Leire Ortiz \\ Fernandez
}

Received: date / Accepted: date

\begin{abstract}
Background: Recently, a home-based rehabilitation system for stroke survivors [1], composed of two linked applications (one for the therapist and another one for the patient), has been introduced. The proposed system has been previously tested on healthy subjects. However, for a fair evaluation, it is necessary to carry out a clinical study considering stroke survivors.

Aim: This work aims at evaluating the home-based rehabilitation system on 10 chronic post-stroke spastic patients.

Methods and Procedures: In this paper, each patient carries out two exercises implying the motion of the spastic upper limb using the home-based rehabilitation system. The impact of the color-based 3D skeletal feedback, guiding the patients during the training, is studied. The Time Variable Replacement (TVR)-based average distance, as well as the average postural angle used in [1], are reported to compare the movement and the posture of the patient with and without showing the feedback proposals, respectively. Furthermore, three different questionnaires, specifically designed for this study, are used to evaluate the user experience of the therapist and the patients.

Outcomes and Results: The postural angle of the patient decreases in the presence of the postural color-based feedback. The reported TVR-based average distance for the simplest exercise also decreases with the use of the motion-based feedback, meaning that the patient follows the proposed motion more closely when guided by the color-based feedback. However, it is not straightforward to analyze the mitigated TVR-based average distance for the second exercise. In general, the answers to the questionnaires of the therapist and the patients are very positive.
\end{abstract}

E. Ghorbel

Tel.: +3524666445731

Fax: +3524666445731

E-mail: enjie.ghorbel@uni.lu

Present address: 29 avenue JFK, Luxembourg city, Luxembourg 
Conclusion and Applications: Overall, the reported results suggest the relevance of the proposed system for home-based rehabilitation of stroke survivors.

What this paper adds? The home-based rehabilitation system [1] for stroke survivors represents a recently introduced low-cost system able to: (1) guide the patients while training with real-time color-based feedback; (2) tailor the exercises according to the patient's conditions; (3) ensure the remote follow-up of the training by the therapist. This study contributes to the literature by clinically evaluating the proposed system on 10 stroke survivors. The experiments suggest the relevance of this system for upper-limb exercising.

\section{Introduction}

Due to the population aging, the number of stroke survivors in Europe is expected to grow by $25 \%$ in the next two decades [2]. Hence, an important involvement of the research community in proposing solutions to enhance their quality of life has been observed. Indeed, stroke survivors are very often affected by motor impairments, directly impacting simple activities in their daily lives.

To maintain and restore these activities, one of the key treatment is rehabilitation. Usually, the clinician prescribes a therapy to the patient in a rehabilitation center [3]. Thus, the patient is monitored and advised by experts in order to improve his functional movements without impairing his health and safety. However, due to the economical burden, this assisted rehabilitation is mainly prescribed for a limited period of time. Then, following this therapy, when the maximum recovery functional level is achieved, a home-based rehabilitation is usually prescribed to the patient [4]. This home-based training consists of a set of exercises chosen by the therapist and prescribed to the patient on a daily basis $[5,6]$. A booklet illustrating and depicting the prescribed exercises is sometimes provided [6] to orient the patient. The advantages of this home-based rehabilitation program are that: (1) they are not costly; and (2) they do not require the physical presence of a therapist.

Nevertheless, an important proportion of stroke survivors do not exercise sufficiently from home and consequently looses gained recovery level and functionality. It can be indirectly caused by the lack of motivation and/or stress. Concretely, this is related to the fact that the clinician cannot control and monitor the home-based training. More specifically, the therapist cannot check: (1) if the patient is exercising; (2) the correctness, frequency and intensity of the exercises while training; and (3) if the patient is risking injuries while training.

To overcome these issues, several computer-based systems have been introduced $[7,8,9,10,11]$. However, most of them do not incorporate a continuous communication between the therapist and the patient. Furthermore, the feedback provided to the patient about the correctness of his/her training is usually difficult to interpret. Recently, in our previous work [1], a novel home-based rehabilitation system based on computer vision tools has been 
introduced. The main novelty of this system is that it provides continuous communication between the therapist and the patient since it is composed of two applications. The first one is designed for enabling the therapist to remotely prescribe personalized exercises, control the training, and adapt the exercises accordingly. The second application enables the patient to load the personalized exercises and train while being guided by two real-time colorbased feedback proposals $[12,13,14,15,16]$. One of the color-based feedback proposal informs the patient about his/her posture, while the other one monitors his/her limb motion. The feedback proposals of interest are respectively called posture feedback and motion feedback. This system has been initially tested on 10 healthy subjects [1]. Nevertheless, these experiments are not sufficient to assess its clinical utility. Thus, in this paper, we propose to report a clinical study of 10 stroke survivors. The experiments have been carried out at Cruces University Hospital (Osakidetza- Basque Health System), with the permanent presence of a physiotherapist. Both feedback proposals have been extensively analyzed. Furthermore, two questionnaires have been prepared in order to collect the respective opinions of the patients and the therapist.

In the following, Section 2 describes the material and methods used to realize this clinical study. Then, Section 3 reports the obtained results and Section 4 discusses and analyses the reported results. Finally, Section 5 concludes this work.

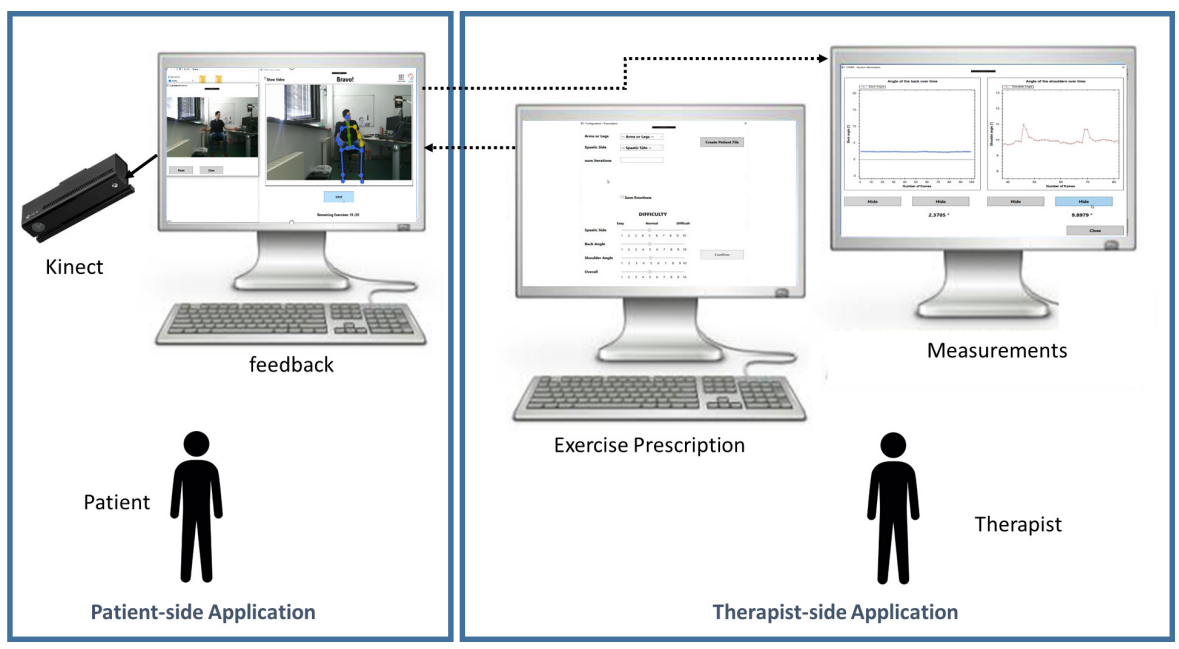

Fig. 1 The architecture of the home-based rehabilitation system proposed in [1]. 


\section{Material and methods}

2.1 Overview of the home-based rehabilitation system [1]

In this section, we briefly present an overview of the home-based rehabilitation system proposed in [1]. The system is constituted of two linked applications, namely the therapist side and the patient side applications, as illustrated in Figure 1.

In the therapist-side application, the therapist can prescribe an exercise tailored to the patient and control his/her progress through relevant measurements describing each training session. Only a simple RGB (Red Green Blue) camera and a standard computer are needed for this application. The RGB camera is only used to record demonstrations of the exercise to be shown to the patient.

The patient-side application is in continuous communication with the therapistside application. For using the patient-side application, only a standard computer and an RGB-D camera (e.g. Kinect) are needed. In fact, with the use of an RGB-D camera, a rough human skeleton of the patient composed of a finite number of joints can be extracted in real-time and used to analyze the patient's movements. The patient-side application interprets the prescriptions sent by the therapist and presents accordingly two different color based-feedback proposals using the captured skeletal information. The first one, called posture feedback, colors the skeleton trunk to guide the posture of the patient: green indicates the correctness while red shows the contrast. The second color-based feedback, termed motion feedback, specifies how correct the motion of the spastic limb is, with respect to the reference motion. Similar to the posture feedback, the motion feedback uses color shades from green to red to guide the patient. The two color-based feedback proposals are visualized on top of the rough patient's skeleton, as shown in Figure 2. The patient observes therefore in real-time his skeleton as well as the color codes that guide him instantly. More details about the computation of these feedback proposals can be found in [1]. Finally, the patient-side application transfers a detailed report to the therapist-side application containing measurements acquired during the training. The different functions of the system are shown in Figure 3.

\subsection{Participants}

In total, 10 patients have participated in this clinical study. All the participants are chronic stroke spastic survivors with different levels of spasticity. Table 1 describes the profile of each patient. Recruitment was performed in outpatients' neurorehabilitation consultation by a physical medicine and rehabilitation doctor following a consecutive sampling method during 3 weeks. All the subjects were written and orally informed and informed consent was signed. All had the possibility to withdraw the consent to participate in the study. 


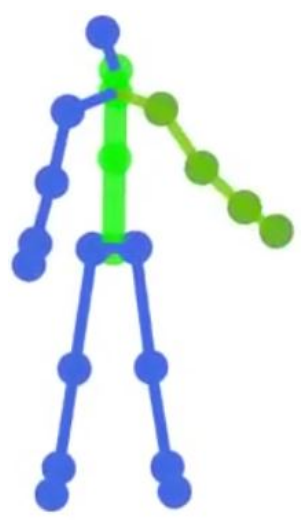

(a)

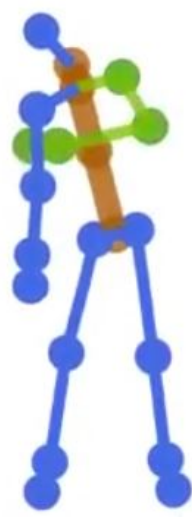

(b)

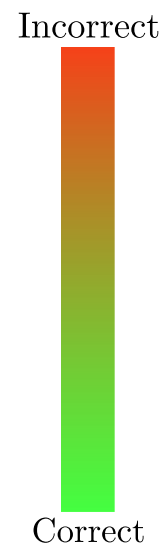

(c)

Fig. 2 Visualization of the color-based feedback proposals [1]. Figure 2(a) shows a correct movement of the spastic limb as well as a correct posture. However, in Figure 2(b) an example where the patient compensates the movement with his back is illustrated. This results in a red coloration of the trunk segment. The movement of the spastic limb is still good in this example resulting in a green color feedback. Figure 2(c) depicts the color range used to indicate the correctness of the movement and the posture.

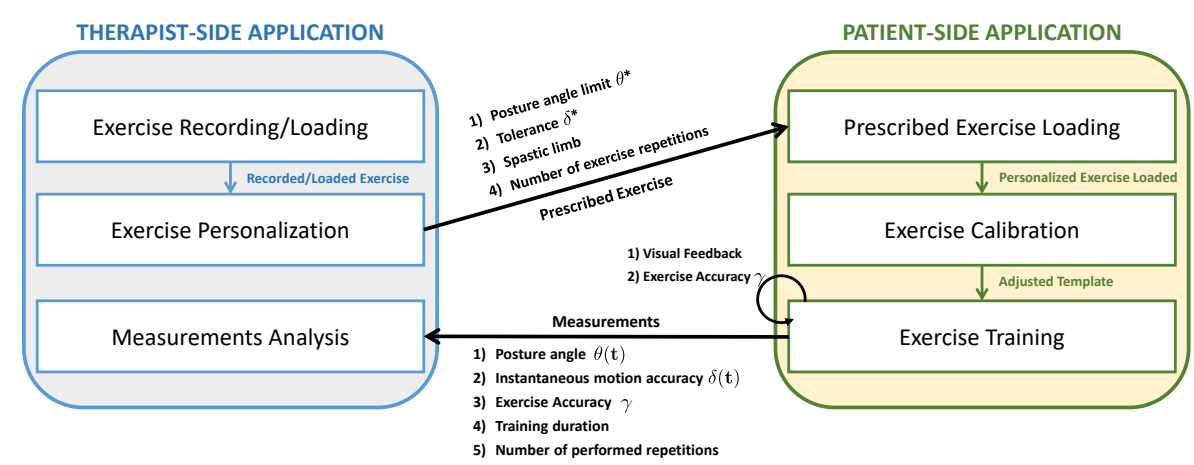

Fig. 3 The functionality of the home-based rehabilitation system [1]

\subsection{Procedures}

The experiments have been completed within five days. During its full duration, a physiotherapist was always present to explain the exercises and intervene if necessary by helping patients in case of severe spasticity. Two different exercises were performed by the 10 patients called scaption and hand to torso, face, and head. The scaption exercise consists in raising the hand in the scapular plane (Exercise 1), while the hand to torso, face, and head exercise, more complex, implies to pass the hand in front of the torso and face before bringing it to the top of the head (Exercise 2). Figure 4 and Figure 5 respectively illustrate Exercise 1 and Exercise 2. The activities were divided into three phases: 


\begin{tabular}{|c|c|c|c|c|c|}
\hline Patient & Gender & Age & Years from stroke (Approx.) & MAS $^{*}$ & Affected side \\
\hline 1 & F & 72 & 9.4 & 3 & Left \\
\hline 2 & F & 75 & 6.6 & 2 & Left \\
\hline 3 & M & 52 & 1 & 1 & Left \\
\hline 4 & M & 76 & 4.6 & 2 & Right \\
\hline 5 & F & 51 & 26 & $2-3$ & Right \\
\hline 6 & F & 40 & 34 & $2-3$ & Right \\
\hline 7 & M & 63 & 8 & $1-2$ & Left \\
\hline 8 & M & 71 & 8.6 & 3 & Left \\
\hline 9 & F & 57 & 6 & 2 & Left \\
\hline 10 & F & 41 & 1.2 & 1 & Right \\
\hline
\end{tabular}

Table 1 Profile of the post-stroke patients (gender, age, years from stroke, MAS*, affected side). *Spasticity level was measured by modified ASHWORTH scale (MAS) which measures the resistance during passive soft-tissue stretching. Scoring range varies from 0 (no increase in muscle tone) to 4 (affected parts are rigid in flexion or extension).
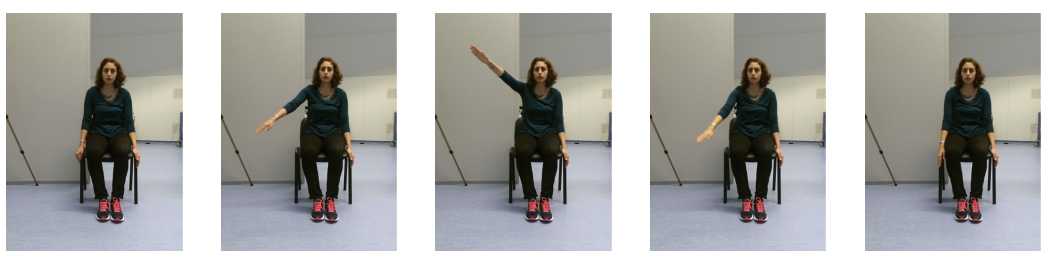

Fig. 4 Illustration of Exercise 1 used for the experiments: the images are organized in chronological order. Better visualized in color.
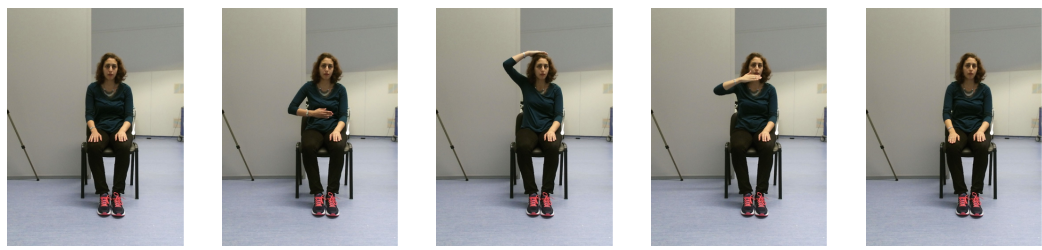

Fig. 5 Illustration of Exercise 2 used for the experiments: the images are organized in chronological order. Better visualized in color.

- Phase 0 (day 1): Before starting exercising, the physiotherapist explains and demonstrates the exercises to the patient. Then, the patient repeats the same exercises with the healthy upper-limb. While performing the exercise, a video containing the same exercise is shown to the patient. As explained in [1], the motion of the healthy limb is captured and is considered as the reference motion. This allows taking into account the anthropometry as well as the moving style of the patient.

- Phase 1 (day 1): During this phase, the patients are asked to run two different exercises with their spastic upper limb. Each exercise is performed 5 times with the spastic hand. In this phase, the color-based feedback proposals are not shown to the patient. Only one day has been dedicated to this phase allowing us to acquire baseline data. 
- Phase 2 (days 2 to 5): For the last phase of the study, the patients are also asked to carry out the same two exercises realized in Phase 0 and Phase 1 with their spastic upper-limb. In contrast to Phase 1 , the feedback is provided to the patient.

For each phase, all patients are asked to perform each exercise 5 times per day. After each training session, the patient and the therapist fill the respective questionnaires presented in Appendix $\mathrm{C}$ and Appendix A. To avoid disturbing the patient, a very brief questionnaire has been prepared. In the last training session, a more extensive questionnaire, reported in Appendix $\mathrm{B}$, is provided to the patient. The 3 questionnaires have been designed in tight collaboration with medical and Human-Computer Interaction experts in order to consider important medical and usability patterns, respectively. They have been specifically prepared for this study and have not been used in a previous one. Most of the questions followed a Likert schema. In this case, the question is formulated as an affirmation (alternating positive and negative affirmations), and the answer can vary on a scale going from 1 to 5 (1 and 5 respectively correspond to completely agree and completely disagree), allowing a simple computation of statistics. To get better explanations, some open questions have also been formulated. The questions have been selected such that different aspects are considered, namely technical problems, utility of feedback, psychology of the patient. The therapist questionnaire gathers a total of 9 Likert questions (with 3 explicative questions if the answers are negative) and 3 open questions. The patient questionnaire designed for each session comprises only 4 Likert questions. Finally, the patient questionnaire designed for the last session is more extensive and is composed of 11 Likert questions and 5 open questions.

Hence, the data acquired in Phase 1 (without feedback) and Phase 2 (with feedback) are compared with respect to the reference data acquired in Phase 0 .

\subsection{Criteria of Evaluation}

Similar to our previous work [1], we use the two criteria of evaluation called TVR-based average distance and postural angle. The TVR-based average distance is a measure that estimates the dissimilarity between two given motions, inspired by the work of [17]. Thus, in our experiments, this measure is computed for each patient between:

- The reference motion and the motion of the spastic upper limb without feedback (Phase 1). The average of this measure is reported and denoted by $d_{\bar{F}}$.

- The reference motion and the motion of the spastic upper limb with feedback (Phase 2). The average of this measure is reported and denoted by $d_{F}$. 
Thus, the more similar to the reference motion the spastic motion is, the lower this distance is and the more it is considered as correct.

The average postural angles without and with feedback are also computed for each patient and respectively denoted by $\theta_{\bar{F}}$ and $\theta_{F}$. As described in [1], the higher the angle is, the worse the posture is.

\section{Results}

In this section, we present the obtained results with 10 post-stroke patients. On the one hand, Figure 6 and Figure 7, respectively, report the obtained average exercise accuracy and postural angle per patient while performing the Exercise 1. On the other hand, Figure 8 and Figure 9, respectively, report the obtained average exercise accuracy and postural angle per patient while performing Exercise 2. It can be noted that usually $d_{F}<d_{\bar{F}}$ for Exercise 1 . Nevertheless, this is not obvious for Exercise 2, where we can observe that $d_{F}$ is not always inferior to $d_{\bar{F}}$. Only the motion of $50 \%$ of the patients (Patient 1 , Patient 4 , Patient 5, Patient 8 and Patient 10) is improved with the use of the feedback. It is also observed that the postural angle is largely reduced in both exercises for almost all the patients. Therefore, one can say that the posture is importantly improved when using the postural feedback; consequently, avoiding musculoskeletal injuries.

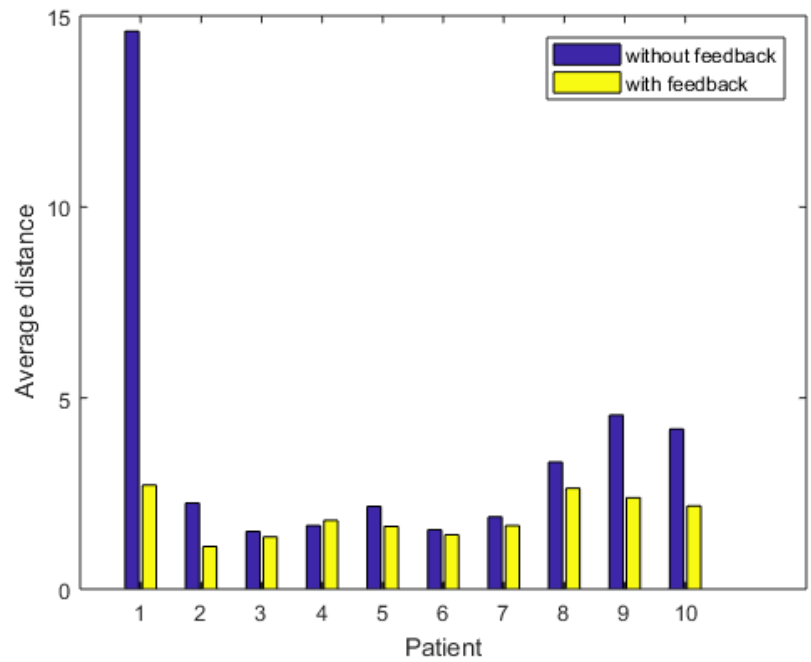

Fig. 6 Average of the distance $d_{\bar{F}}$ and $d_{F}$ per patient obtained for Exercise 1 (respectively without and with feedback). Better visualized in color. 




Fig. 7 Average of the postural angle $\theta_{\bar{F}}$ and $\theta_{F}$ (in degrees) per patient during Exercise 1 (respectively without and with feedback). Better visualized in color.

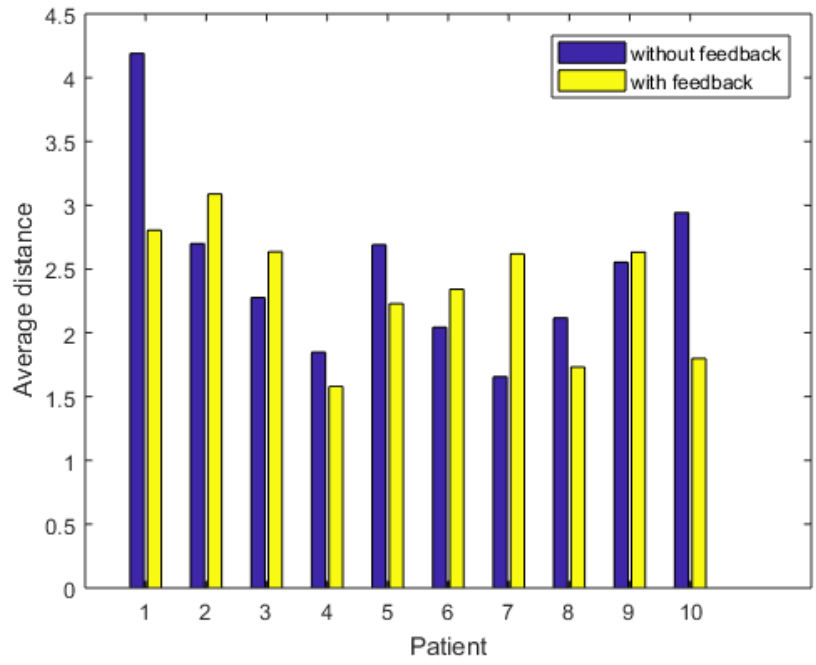

Fig. 8 Average of the distance $d_{\bar{F}}$ and $d_{F}$ per patient obtained for Exercise 2 (respectively without and with feedback). Better visualized in color.

\section{Discussion}

This paper aims at clinically evaluating the different components of the proposed home-based rehabilitation system for stroke survivors. 


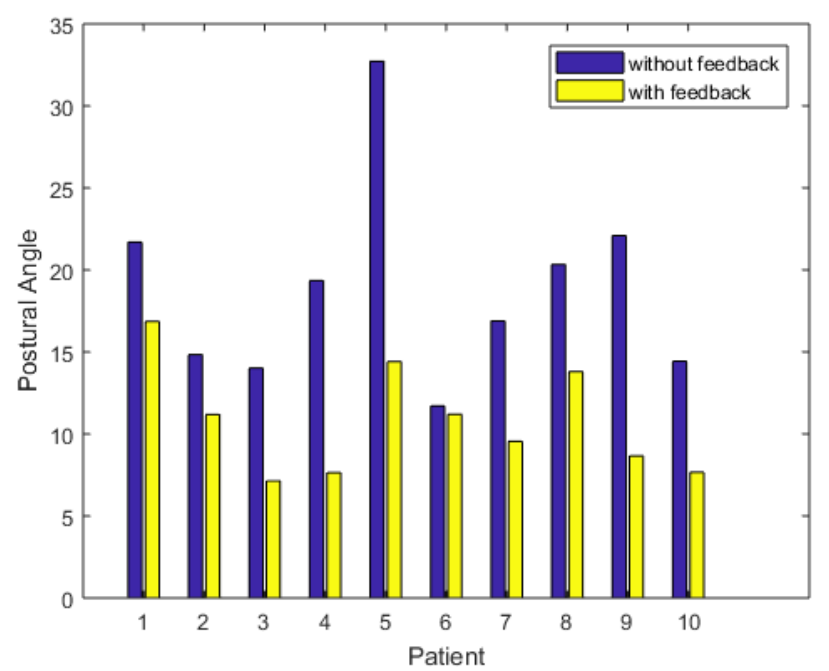

Fig. 9 Average of the postural angle $\theta_{\bar{F}}$ and $\theta_{F}$ (in degrees) per patient during Exercise 2 (respectively without and with feedback). Better visualized in color.

Postural feedback The reported results show the utility of the postural feedback provided by the home-based rehabilitation system. Indeed, as mentioned in Section 3, for both exercises, the postural angle is widely reduced. $44 \%$ of the participating patients spontaneously said that the strength of this system is its posture correction. This feature can be seen as the most important one since it allows the prevention of musculoskeletal injuries and spasticity increase resulting from movement compensation.

Motion-based feedback In contrast to the postural feedback, the relevance of the motion-based feedback is not as clear. As mentioned earlier, while in Exercise 1, we can observe a clear improvement of the movement, this is less evident to claim in Exercise 2. This can be explained by the fact that Exercise 1 is easier to execute than Exercise 2. A more complex exercise is usually more difficult to reproduce identically than a simple one. Despite that, $100 \%$ of the patients completely agree that the color-based feedback is useful. Similarly, the physiotherapist also completely agrees with that in $100 \%$ of the training sessions. Notwithstanding, it is difficult to say if this system has a positive impact on the patient's recovery since it has been tested within a limited period of 5 days. This is confirmed by the nuanced perception of the patients: $33.3 \%$ completely agree, $22.2 \%$ agree, $33.3 \%$ do not agree or disagree and $11.1 \%$ completely disagree that their condition has improved since they are using the application. In contrast, the therapist confirmed that, in $78.3 \%$ of the cases, the application helped the patient improving his controlled movement. 
Measurement report Thanks to the questionnaire addressed to the therapist, we can note that the measurement report received by the therapist is relevant. In $59.4 \%$ of the cases, the therapist finds that the measurements reflect the training of the patient, does not have any opinion about that in $29.7 \%$ of the cases, and thinks that the measurements are not relevant in $10.8 \%$ of the cases.

Reliability and simplicity of the system The proposed system has the advantage to be reliable. The therapist reports that in $97.3 \%$ of the tests, the system did not generate bugs. $88.89 \%$ of the post-stroke patients find that the capture of the movement is accurate and well reproduced on the screen. They state, in $97.1 \%$ of the cases, that the system is easy to use, while the therapist confirms this, in $100 \%$ of the cases. Finally, all the patients describe their experience as very positive or good.

Safety of the application $100 \%$ of the patients feel in security while training with the application. They were sitting on a chair and working on the upper limb such that they felt comfortable and without any risk of falling. However, due to visual or cognitive problems or the inability that few patients have to make painless active movements, the physiotherapist agrees only in $79.3 \%$ of the cases that the patient can use the application alone at home in security; thus, avoiding the generation of abnormal movements, the exaggeration of compensatory patterns or/and pain. For this reason, the permanent presence of the therapist during the first sessions is of high importance, allowing the reinforcement of the correct movements while using system. This is also confirmed by patients' answers: while $88.9 \%$ would like to continue using it in the presence of the therapist, only $33.3 \%$ feel ready to use it alone.

Psychology of the patient According to the questionnaires, the application has a positive impact on the psychology of the patients. In $100 \%$ of the cases, the patients completely disagree that the training sessions are boring and that the exercises are hard to perform. Moreover, in $88.9 \%$ of the cases, they felt more motivated to exercise since they are using the application. On the other side, the therapist finds that patients were feeling comfortable with the application in $71.3 \%$ of the cases.

\section{Conclusion}

In this paper, the home-based rehabilitation system proposed in [1] has been tested on 10 chronic post-stroke spastic patients. The results show that the color-feedback proposals improve the posture of the patients and can help to enhance the motion in the case of simple exercises. In general, the application can be considered as reliable, simple to use, and positively impacting the psychology of the patients. In this work, since the clinical study has been held within five days, it remains difficult to analyze the long-term impact of using such an application. In future work, it would be interesting to evaluate the 
system within a longer period and with more patients. Besides, since the clinical study has focused only on upper limbs, it would be valuable to test the same concept on the lower limbs.

\section{Compliance with Ethical Declarations}

Conflict of interest

The authors declare that they have no conflict of interest.

Funding

This work has been funded by the European Union's Horizon 2020 research and innovation project STARR under grant agreement No.689947.

\section{Ethical approval}

All procedures performed in studies involving human participants were in accordance with the ethical standards of the institutional and/or national research committee and with the 1964 Helsinki declaration and its later amendments or comparable ethical standards.

\section{References}

1. R. Baptista, E. Ghorbel, A. E. R. Shabayek, F. Moissenet, D. Aouada, A. Douchet, M. André, J. Pager, S. Bouilland, Home self-training: Visual feedback for assisting physical activity for stroke survivors, Computer methods and programs in biomedicine 176 (2019) 111-120.

2. E. Emmett, E. Stevens, Y. Cang, C. McKevitt, CDA. Wolfe, The Burden of Stroke in Europe Report. King's College London for the Stroke Alliance for Europe (SAFE): 2017 (2017).

3. J. M. Veerbeek, E. van Wegen, R. van Peppen, P. J. van der Wees, E. Hendriks, M. Rietberg, G. Kwakkel, What is the evidence for physical therapy poststroke? a systematic review and meta-analysis, PloS one (2014).

4. P. Langhorne, G. Taylor, G. Murray, M. Dennis, C. Anderson, E. BautzHolter, P. Dey, B. Indredavik, N. Mayo, M. Power, et al., Early supported discharge services for stroke patients: a meta-analysis of individual patients' data, The Lancet (2005).

5. D. D. N. Natta, E. Alagnide, G. T. Kpadonou, G. G. Stoquart, C. Detrembleur, T. M. Lejeune, Feasibility of a self-rehabilitation program for the upper limb for stroke patients in benin, Annals of Physical and Rehabilitation Medicine 58 (2015) 322 - 325 . 
6. C. Bonnyaud, P. Gallien, P. Decavel, P. Marque, C. Aymard, F. Pellas, M.E. Isner, F. C. Boyer, F. Muller, J.-C. Daviet, P. Dehail, B. Perrouin-Verbe, N. Bayle, E. Coudeyre, D. Perennou, I. Laffont, J. Ropers, N.-Y. DomingoSaidji, D. Bensmail, N. Roche, Effects of a 6-month self-rehabilitation programme in addition to botulinum toxin injections and conventional physiotherapy on limitations of patients with spastic hemiparesis following stroke (adju-tox): protocol study for a randomised controlled, investigator blinded study, BMJ Open 8 (2018).

7. S. Simmons, R. McCrindle, M. Sperrin, A. Smith, Prescription software for recovery and rehabilitation using microsoft kinect, in: Pervasive Computing Technologies for Healthcare (PervasiveHealth), 2013 7th International Conference on, IEEE, pp. 323-326.

8. F. Spyridonis, J. Gawronski, G. Ghinea, A. O. Frank, An interactive 3-d application for pain management: Results from a pilot study in spinal cord injury rehabilitation, Computer methods and programs in biomedicine 108 (2012) 356-366.

9. J. M. I. Zannatha, A. J. M. Tamayo, A. D. G. Sánchez, J. E. L. Delgado, L. E. R. Cheu, W. A. S. Arévalo, Development of a system based on $3 \mathrm{~d}$ vision, interactive virtual environments, ergonometric signals and a humanoid for stroke rehabilitation, Computer Methods and Programs in Biomedicine 112 (2013) 239 - 249. SI: Computer Assisted Tools for Medical Robotics.

10. S. Spasojević, N. V. Ilić, A. Rodić, J. Santos-Victor, Kinect-based application for progress monitoring of the stroke patients, in: Proceedings of IcETRAN conference, vol. ROI2, volume 6, pp. 1-5.

11. M. Trombetta, P. P. B. Henrique, M. R. Brum, E. L. Colussi, A. C. B. De Marchi, R. Rieder, Motion rehab ave 3d: a vr-based exergame for poststroke rehabilitation, Computer methods and programs in biomedicine 151 (2017) 15-20.

12. M. Antunes, R. Baptista, G. Demisse, D. Aouada, B. Ottersten, Visual and human-interpretable feedback for assisting physical activity, in: European Conference on Computer Vision (ECCV) Workshop on Assistive Computer Vision and Robotics Amsterdam.

13. R. Baptista, M. Antunes, A. E. R. Shabayek, D. Aouada, B. Ottersten, Flexible feedback system for posture monitoring and correction, in: IEEE International Conference on Image Information Processing (ICIIP).

14. R. Baptista, E. Ghorbel, A. E. R. Shabayek, D. Aouada, B. Ottersten, Key-skeleton based feedback tool for assisting physical activity, in: 2018 Zooming Innovation in Consumer Technologies Conference (ZINC), IEEE, pp. $175-176$.

15. M. Antunes, D. Aouada, G. Demisse, Physical activity feedback, Patent WO2017207802A1 (2017).

16. A. Shabayek, R. Baptista, K. Papadopoulos, G. Demisse, O. Oyedotun, M. Antunes, D. Aouada, B. Ottersten, M. Anastassova, M. Boukallel, S. Panëels, G. Randall, M. Andre, A. Douchet, S. Bouilland, L. Ortiz Fernandez, STARR - Decision SupporT and self-mAnagement system for 
stRoke survivoRs Vision based Rehabilitation System, European Project Space on Networks, Systems and Technologies - Volume 1: EPS Porto 2017 (2017).

17. E. Ghorbel, R. Boutteau, J. Boonaert, X. Savatier, S. Lecoeuche, Kinematic spline curves: A temporal invariant descriptor for fast action recognition, Image and Vision Computing (2018). 